TRANSACTIONS OF THE

AMERICAN MATHEMATICAL SOCIETY

Volume 352 , Number 4, Pages 1935-1946

S 0002-9947(99)02419-8

Article electronically published on December 14, 1999

\title{
SIMPLE AND SEMISIMPLE LIE ALGEBRAS AND CODIMENSION GROWTH
}

\author{
ANTONIO GIAMBRUNO, AMITAI REGEV, AND MICHAIL V. ZAICEV
}

\begin{abstract}
We study the exponential growth of the codimensions $c_{n}^{L}(B)$ of a finite dimensional Lie algebra $B$ over a field of characteristic zero. In the case when $B$ is semisimple we show that $\lim _{n \rightarrow \infty} \sqrt[n]{c_{n}^{L}(B)}$ exists and, when $F$ is algebraically closed, is equal to the dimension of the largest simple summand of $B$. As a result we characterize central-simplicity: $B$ is central simple if and only if $\operatorname{dim} B=\lim _{n \rightarrow \infty} \sqrt[n]{c_{n}^{L}(B)}$.
\end{abstract}

\section{INTRODUCTION AND THE MAIN RESUlts}

Let $A$ be an associative P.I. algebra. The identities of $A, \operatorname{Id}(A)$, form a $T$-ideal in the free associative algebra $F\langle X\rangle=F\left\langle x_{1}, x_{2}, \ldots\right\rangle: I d(A) \subseteq F\langle X\rangle . V_{n}$ are the multilinear polynomials in $x_{1}, \ldots, x_{n}, F S_{n}$ is the group algebra of the symmetric group $S_{n}$, and $\sigma \longleftrightarrow x_{\sigma(1)} \cdots x_{\sigma(n)}$ gives an isomorphism of $F S_{n}$ and $V_{n}$ as left $F S_{n}$ modules. The cocharacters $\chi_{n}(A)$ and the codimensions $c_{n}(A)$ of $A$ are defined via the quotient modules $V_{n} /\left(\operatorname{Id}(A) \cap V_{n}\right)$, [R1], R2].

The generalization of these invariants to Lie algebras was first considered by Volichenko $[\mathrm{V}]$. Let $B$ be a Lie algebra. Its identities $I d^{L}(B)$ are elements of the free Lie algebra $L\langle X\rangle$. Let $V_{n}^{L} \subseteq L\langle X\rangle$ denote the multilinear Lie polynomials in $x_{1}, \ldots, x_{n}$; then, in complete analogy to the associative case, $V_{n}^{L} /\left(I^{L}(B) \cap V_{n}^{L}\right)$ defines the Lie cocharacter $\chi_{n}^{L}(B)$ with the corresponding Lie codimension $c_{n}^{L}(B)=$ $\operatorname{deg} \chi_{n}^{L}(B)$. We write $\chi_{n}^{L}(B)=\sum_{\lambda \vdash n} m_{\lambda}^{L}(B) \chi_{\lambda}$ (see 2.4 for generalizations).

In the associative case, the basic property of the codimensions is that they are exponentially bounded:

Theorem [R1]. Let $A$ be an associative P.I. algebra. Then there exist $k>0$ and $\alpha \geq 1$ such that

$$
c_{n}(A) \leq k \cdot \alpha^{n}
$$

for all $n$.

In this paper we study the Lie codimensions of a finite dimensional Lie algebra over a field $F$ of characteristic zero. It was shown by Volichenko $[\mathrm{V}]$ that, in general, Lie codimensions need not be exponentially bounded. However, by the

Received by the editors November 27, 1997.

2000 Mathematics Subject Classification. Primary 17B01, 17B20, 16R10, Secondary 20C30, $17 \mathrm{C} 05$.

Key words and phrases. Lie algebras, polynomial identities, codimensions.

The first author was partially supported by MURST and CNR of Italy.

The second author was partially supported by NSF Grant No. DMS-94-01197.

The third author was partially supported by RFFI grants 96-01-00146 and 96-15-96050. 
Ado-Iwasawa theorem (see $[\mathrm{B}, 6.2 .3]$ ) every finite dimensional Lie algebra $B$ over $F$ has a faithful finite dimensional representation. It follows that $B \subseteq A^{(-)}$, where $A$ is an associative finite dimensional algebra (hence is P.I.) and $A^{(-)}$is its Lie structure. As we show below, this implies that $\left\{c_{n}^{L}(B)\right\}$ is also exponentially bounded (see [B] ).

In general, $\left\{c_{n}^{L}(B)\right\}$ seems to be rather complicated, and even its asymptotic behavior is hard to describe. If $c_{n}^{L}(B)$ is exponentially bounded, it seems reasonable to conjecture that for some constants $a, g$ and $\alpha, c_{n}^{L}(B) \underset{n \rightarrow \infty}{\stackrel{\sim}{\rightarrow}} a \cdot n^{g} \cdot \alpha^{n}$. Similar remarks hold in the associative case.

To capture the exponential growth of $\left\{c_{n}^{L}(B)\right\}$ we introduce

1.1 Definition. Let $B$ be a finite dimensional Lie algebra. Define

$$
I \overline{n v}^{L}(B)=\limsup _{n \rightarrow \infty} \sqrt[n]{c_{n}^{L}(B)}
$$

and

$$
\underline{\operatorname{Inv}}^{L}(B)=\liminf _{n \rightarrow \infty} \sqrt[n]{c_{n}^{L}(B)}
$$

In case of equality, denote

$$
\operatorname{Inv}{ }^{L}(B)=\underline{\operatorname{Inv}}^{L}(B)=\operatorname{Inv} v^{L}(B) .
$$

Here we study these invariants when $B$ is a simple or semisimple finite dimensional Lie algebra over a field $F, \operatorname{char}(F)=0$. The main results of this paper are:

1.2 Theorem (7.3 below). Let $F$ be algebraically closed. Then $B$ is simple if and only if $\operatorname{Inv} \bar{v}^{L}(B)=\underline{\operatorname{Inv}}^{L}(B)$ and $\operatorname{Inv} v^{L}(B)=\operatorname{dim} B$. Moreover, if $B$ is not simple then $\operatorname{Inv}^{L}(B) \leq \operatorname{dim} B-1$.

1.3 Theorem (7.1 below). Let $F$ be algebraically closed, $B=\bigoplus_{i} B_{i}, B_{i}$ simple. Let $m_{0}=\max _{i}\left\{\operatorname{dim} B_{i}\right\}$. Then

$$
\operatorname{Inv}{ }^{L}(B)=\underline{\operatorname{Inv}}^{L}(B)=\operatorname{Inv}^{L}(B)=m_{0} .
$$

1.4 Theorem (8.1 below). B is a central simple Lie algebra if and only if

$$
\operatorname{Inv}{ }^{L}(B)=\underline{\operatorname{Inv}}^{L}(B)=\operatorname{Inv} v^{L}(B)=\operatorname{dim} B .
$$

1.5 Theorem (8.2 below). If $B$ is simple, then $\operatorname{Inv}^{L}(B)$ exists, is a positive integer which divides $\operatorname{dim} B$, and

$$
\operatorname{dim} B=\left(\operatorname{Inv}^{L}(B)\right) \cdot[\operatorname{centroid}(B): F] .
$$

1.6 Theorem (8.3 below). Let $B$ be semisimple and let $\bar{F}$ be the algebraic closure of $F$. It is known that $\bar{B}=B \otimes_{F} \bar{F}$ is also semisimple over $\bar{F}$. Let $\bar{B}=\bigoplus_{i} \bar{B}_{i}$, $\bar{B}_{i}$ simple over $\bar{F}$. Let $m_{0}=\max _{i}\left\{\operatorname{dim}_{\bar{F}} \bar{B}_{i}\right\} . \quad$ Then $\operatorname{Inv} \bar{n}^{L}(B)=\underline{\operatorname{Inv}}^{L}(B)=$ $\operatorname{Inv}^{L}(B)=m_{0}\left(=\operatorname{Inv}^{L}(\bar{B})\right)$.

1.7 Theorem (8.4 below). If $B$ is not simple, then $\operatorname{Inv}^{L}(B) \leq \operatorname{dim} B-1$.

1.8 Theorem (8.5 below). Let $\widetilde{B}=B \otimes_{F} F\left[t, t^{-1}\right]+\langle z\rangle$ be a non-twisted affine Kac-Moody algebra corresponding to $B$. If $B$ is semisimple, then $\operatorname{Inv}^{L}(\widetilde{B})$ exists and $\operatorname{Inv}^{L}(\widetilde{B})=\operatorname{Inv}^{L}(B)$ (hence is an integer by 1.6). 
For Jordan algebras $M$ we define $\operatorname{Inv} v^{J}(M)$ similarly. In section 10 we calculate $\operatorname{Inv}^{J}(M)$ in some special cases where $M$ are Jordan matrix algebras.

The basic technique for proving that $\operatorname{Inv}^{L}(B)=\alpha$ is to find constants $a_{1}, a_{2} \geq 0$, $g_{1}$ and $g_{2}$ such that

$$
a_{2} n^{g_{2}} \alpha^{n} \leq c_{n}^{L}(B) \leq a_{1} n^{g_{1}} \alpha^{n}
$$

for all $n$. Both bounds follow from studying the cocharacters $\chi_{n}^{L}(B)$ : upper bounds follow height-restrictions on the Young diagrams in $\chi_{n}^{L}(B)$, as well as from the polynomial bounds on their multiplicities [BR]. An important tool for constructing the lower bounds is a theorem of Razmyslov [Ra 12.1], which implies the existence in $\chi_{n}^{L}(B)$ of a certain rectangular diagram of arbitrary length.

\section{The VARious CODIMENSIONS}

Here we look more closely at Lie codimensions and cocharacters, as well as at some generalizations.

Recall from $\S 1$ that $c_{n}^{L}(B)=\operatorname{deg} \chi_{n}^{L}(B)$, where $\chi_{n}^{L}(B)$ is the $S_{n}$-character of the left $F S_{n}$ module $V_{n}^{L} /\left(I d^{L}(B) \cap V_{n}^{L}\right)$. Denote $\chi_{n}^{L}(B)=\sum_{\lambda \vdash n} m_{\lambda}^{L}(B) \cdot \chi_{\lambda}$. Here $\lambda$ is a partition of $n, \chi_{\lambda}$ the corresponding $S_{n}$-irreducible character, with multiplicity $m_{\lambda}^{L}(B)$ in $\chi_{n}^{L}(B)$.

Call $\left[x_{1},\left[x_{2}, \ldots,\left[x_{n-2},\left[x_{n-1}, x_{n}\right]\right] \ldots\right]\right]=\left[x_{1}, \ldots, x_{n}\right]$ "right normed Lie monomial". It easily follows from the Jacobi-identity that

$$
V_{n+1}^{L}=V_{n+1}^{L}\left(x_{1}, \ldots, x_{n}, x_{0}\right)=\operatorname{Span}_{F}\left\{\left[x_{\sigma(1)}, \ldots, x_{\sigma(n)}, x_{0}\right] \mid \sigma \in S_{n}\right\} .
$$

Both $V_{n}$ and $V_{n+1}^{L}$ are left $F S_{n}$ modules in an obvious way, and the map

$$
\varphi: x_{\sigma(1)} \cdots x_{\sigma(n)} \rightarrow\left[x_{\sigma(1)}, \ldots, x_{\sigma(n)}, x_{0}\right]
$$

is an $F S_{n}$-module-isomorphism $V_{n} \simeq_{F S_{n}} V_{n+1}^{L}$. In particular, $\operatorname{dim} V_{n+1}^{L}=n$ !. Given $f \in V_{n}$, denote

$$
\varphi(f)=f^{L}=f^{L}\left(x_{1}, \ldots, x_{n}, x_{0}\right) \in V_{n+1}^{L} .
$$

Recall the "ad" map for Lie algebras: $a d: B \rightarrow a d B \subseteq \operatorname{End}_{F}(B)$. We say that the associative polynomial $f\left(x_{1}, \ldots, x_{n}\right) \in F\langle X\rangle$ is an identity of $a d B$ if $f\left(a d b_{1}, \ldots, a d b_{n}\right)=0$ for all $b_{1}, \ldots, b_{n} \in B$ (i.e. $f$ is an identity of the pair $\left.\left(a d B, \operatorname{End}_{F}(B)\right)[\mathrm{Ra}]\right)$.

2.1 Lemma. Let $B$ be a Lie algebra and let $f\left(x_{1}, \ldots, x_{n}\right) \in V_{n}$. Then $f$ is an identity of $a d B$ if and only if $f^{L}\left(x_{1}, \ldots, x_{n}, x_{0}\right)$ is an identity of $L$.

Proof. This lemma easily follows from the equality

$$
f\left(a d x_{1}, \ldots, a d x_{n}\right)\left(x_{0}\right)=f^{L}\left(x_{1}, \ldots, x_{n}, x_{0}\right) .
$$

Recall the decomposition $F S_{n}=\bigoplus_{\lambda \vdash n} I_{\lambda}$, where the $I_{\lambda}$ are minimal two-sided ideals.

2.2 Lemma. Let $B$ be a Lie algebra. Let $\lambda \vdash n$ with the corresponding two sided ideal $I_{\lambda} \subseteq F S_{n}$. Let $f\left(x_{1}, \ldots, x_{n}\right) \in I_{\lambda}$ be such that $f^{L}\left(x_{1}, \ldots, x_{n}, x_{0}\right)$ is a nonidentity of $B$ (or equivalently, $f$ is a non-identity of adB). Then $c_{n+1}^{L}(B) \geq d_{\lambda}$ (where $\operatorname{dim} I_{\lambda}=d_{\lambda}^{2}$ ). 
Proof. The $F S_{n}$ left module isomorphism $V_{n} \cong V_{n+1}^{L}$ restricts to $F S_{n} \cdot f \cong F S_{n} \cdot f^{L}$. Since $f$ is a non-identity, $F S_{n} \cdot f$ contains a minimal left ideal $0 \neq J_{\lambda} \subseteq I_{\lambda}$, all of whose non-zero elements are non-identities of $a d B$. Hence, by $2.1, J_{\lambda}^{L} \cap I d^{L}(B)=0$, where $J_{\lambda}^{L}=\varphi\left(J_{\lambda}\right)$. But $\operatorname{dim} J_{\lambda}^{L}=\operatorname{dim} J_{\lambda}=d_{\lambda}$; hence $c_{n+1}^{L}(B) \geq d_{\lambda}$.

We shall need the following extension of 2.2 :

2.3 Lemma. Let $B$ be a Lie algebra, $\lambda \vdash n, f\left(x_{1}, \ldots, x_{n}\right) \in I_{\lambda}$, and $0 \leq q \in \mathbb{Z}$, such that $f\left(x_{1}, \ldots, x_{n}\right) x_{n+1} \cdots x_{n+q}$ is a non-identity of adB. Then $c_{n+q+1}^{L}(B) \geq$ $d_{\lambda}$.

Proof. Let $f(x) \in J_{\lambda} \subseteq I_{\lambda}, J_{\lambda}$ as in the proof of 2.2. Then

$$
f\left(x_{1}, \ldots, x_{n}\right) x_{n+1} \cdots x_{n+q} \in F S_{n+q} J_{\lambda} .
$$

By the branching rule for $F S_{n}, F S_{n+q} J_{\lambda}$ is a sum of irreducible left ideals $J_{\mu}$ (in $F S_{n+q}$ ), for partitions $\lambda \subseteq \mu \vdash n+q$. By irreducibility, it follows that there exist such $\mu$ and a left ideal $J_{\mu}$ all of whose non-zero elements are non-identities of $a d B$. As in 2.2 , this implies that $c_{n+q+1}^{L}(B) \geq d_{\mu}$. Finally, again by the branching rule, $d_{\mu} \geq d_{\lambda}$.

Cocharacters and codimensions of other algebraic structures can be defined analogously. For example, we discuss these invariants for $S$-algebras which are embedded in the associative P.I. algebras.

2.4 $S$-algebras. Let $A$ be an associative algebra over a field $F$. Let $\alpha, \beta \in F$ and let $*$ be the operation on $A$ defined by setting $a * b=\alpha a b+\beta b a$, for $a, b \in A$. Then $*$ defines a structure of a (not necessarily associative) algebra on the underlying vector space of $A$. We shall assume that $\alpha$ and $\beta$ are not both zero, and we shall say that $A(+, *)$ is an $S$-algebra. The following are the two most significant examples: if $[a, b]=a b-b a$, the bracket operation [, ] defines a structure of Lie algebra on $A$, denoted by $A^{(-)}$; also, we may regard $A$ as a special Jordan algebra under the operation $\{a, b\}=a b+b a$ and denoted it by $A^{(+)}$. If $B$ is a subspace of $A$ invariant under $*$, we shall say that $B$ is an $S$-subalgebra of $A$.

Let $F_{S}\langle X\rangle$ be the $S$-subalgebra of $F\langle X\rangle$ generated by $X ; F_{S}\langle X\rangle$ is the free $S$-algebra on $X$. Let $V_{n}^{S}$ be the space of multilinear $S$-polynomials in $x_{1}, \ldots, x_{n}$. If $f\left(x_{1}, \ldots, x_{n}\right) \in V_{n}^{S}$ is a multilinear $S$-polynomial and $\sigma \in S_{n}$, then

$$
\sigma f\left(x_{1}, \ldots, x_{n}\right)=f\left(x_{\sigma(1)}, \ldots, x_{\sigma(n)}\right)
$$

induces a structure of $S_{n}$-module on $V_{n}^{S}$. Let $I d^{S}(B) \subseteq F_{S}\langle X\rangle$ be the $T$-ideal of $S$-identities of $B$. Then $V_{n}^{S} \cap I d^{S}(B)$ is invariant under the $S_{n}$-action, and we denote by $\chi_{n}^{S}(B)$ the character of the left $S_{n}$-module $\frac{V_{n}^{S}}{V_{n}^{S} I d^{S}(B)} ; \chi_{n}^{S}(B)$ is the $n$-th $S$-cocharacter of $B$ and $c_{n}^{S}(B)=\operatorname{deg} \chi_{n}^{S}(B)$ the $n$-th $S$-codimension of $B$. Let $\lambda \vdash n$ with $\chi_{\lambda}$ the corresponding irreducible $S_{n}$-character; then $\chi_{n}^{S}(B)=\sum_{\lambda \vdash n} m_{\lambda}^{S}(B) \chi_{\lambda}$, for some multiplicities $m_{\lambda}^{S}(B)$.

In section 3 we shall observe some obvious relations between these invariants.

\section{Comparison Between the Various COCHARACTERS}

Here we study the relations between the various cocharacters. Those of associative algebras yield upper bounds, bounds that imply: 
3.1 Theorem. Let $A$ be an associative algebra, $B \subseteq A^{(-)}$a Lie subalgebra (or more generally, $B \subseteq A(+, *)$ an $S$-algebra). Let $\operatorname{dim} B=m$. Write $\chi_{n}^{L}(B)=$ $\sum_{\lambda \vdash n} m_{\lambda}^{L}(B) \chi_{\lambda}$ and $c_{n}^{L}(B)=\operatorname{deg} \chi_{n}^{L}(B)$. Then:

1. There exists $r_{1}$, such that for all $n$ and all $\lambda \vdash n, m_{\lambda}^{L}(B) \leq n^{r_{1}}$.

2. There exist constants $C, r_{2}$ such that for all $n, c_{n}^{L}(B) \leq C \cdot n^{r_{2}} \cdot m^{n}$.

The proof will follow from

3.2 Lemma. As in 2.4, let $B \subseteq A(+, *)$ be an $S$-subalgebra of the associative P.I. algebra $A$. Write $\chi_{n}(A)=\sum_{\lambda \vdash n} m_{\lambda}(A) \chi_{\lambda}$ and $\chi_{n}^{S}(B)=\sum_{\lambda \vdash n} m_{\lambda}^{S}(B) \chi_{\lambda}$.

Then $m_{\lambda}^{S}(B) \leq m_{\lambda}(A)$ for all $\lambda$, i.e.

$$
\chi_{n}^{S}(B) \leq \chi_{n}(A) .
$$

Proof. Let $Q \subseteq F\langle X\rangle$ be the ideal of associative polynomials vanishing when evaluated in $B$, i.e.,

$$
Q=\left\{f\left(x_{1}, \ldots, x_{k}\right) \in F\langle X\rangle \mid f\left(b_{1}, \ldots, b_{k}\right)=0 \text {, for all } b_{i} \in B\right\} .
$$

Since $V_{n} \cap Q$ is invariant under the $S_{n}$ action, $\frac{V_{n}}{V_{n} \cap Q}$ is a left $F S_{n}$-module and we denote by $\chi_{n}^{a s}(B)$ the corresponding $S_{n}$ character. We shall prove that for all $n$

$$
\chi_{n}^{S}(B) \leq \chi_{n}^{a s}(B) \leq \chi_{n}(A) .
$$

Since $V_{n}^{S} \cap I d^{S}(B)=V_{n}^{S} \cap Q \subseteq V_{n} \cap Q$, there is a natural monomorphism of $F S_{n^{-}}$ modules $\frac{V_{n}^{S}}{V_{n}^{S} \cap d^{S}(B)} \rightarrow \frac{V_{n}}{V_{n} \cap Q}$. Hence $\chi_{n}^{S}(B) \leq \chi_{n}^{a s}(B)$. Also, since $\operatorname{Id}(A) \subseteq Q$, it follows that there is a natural epimorphism of $S_{n}$-modules $\frac{V_{n}}{V_{n} \cap I d(A)} \rightarrow \frac{V_{n}}{V_{n} \cap Q}$. Hence $\chi_{n}^{a s}(B) \leq \chi_{n}(A)$, and the lemma is proved.

3.3 Corollary. If $\chi_{n}^{S}(B)=\sum_{\lambda \vdash n} m_{\lambda}(B) \chi_{\lambda}$ and $\chi_{n}^{a s}(B)=\sum_{\lambda \vdash n} m_{\lambda}^{\prime}(B) \chi_{\lambda}$, then for all $\lambda \vdash n, m_{\lambda}(B) \leq|\lambda|^{r}$ and $m_{\lambda}^{\prime}(B) \leq|\lambda|^{r}$, for some fixed $r$. This proves the first part of 3.1 .

Proof. This follows from the previous theorem since by [BR, Theorem 16] the multiplicities in the $n$-th cocharacter of an associative P.I.-algebra are polynomially bounded.

Recall the notation

$$
H(k, l ; n)=\left\{\lambda=\left(\lambda_{1}, \lambda_{2}, \ldots\right) \vdash n \mid \lambda_{k+1} \leq l\right\} .
$$

3.4 Lemma. Let $B \subseteq A(+, *), \operatorname{dim} B=m$, and let $\chi_{n}^{S}(A)$ and $\chi_{n}^{a s}(B)$ be as in 3.2. Then

$$
\chi_{n}^{S}(B) \leq \chi_{n}^{a s}(B)=\sum_{\lambda \in H(m, 0 ; n)} m_{\lambda}^{\prime}(B) \chi_{\lambda}
$$

(and by $3.3 m_{\lambda}^{\prime}(B) \leq|\lambda|^{r}$ for some fixed $r$ ).

Proof. Let $f=f\left(x_{1}, \ldots, x_{m+1}, y_{1}, y_{2}, \ldots\right)$ be multilinear and alternating in $x_{1}$, $\ldots, x_{m+1}$. Since $\operatorname{dim} B=m, f=0$ is an identity for $B: f \in Q$.

Let $\lambda=\left(\lambda_{1}, \lambda_{2}, \ldots\right) \vdash n$ with $\lambda_{m+1} \geqq 0$ and $f(x) \in I_{\lambda}$. Then $f$ is a linear combination of polynomials, each with an alternating subset of (at least) $m+1$ variables; hence $f \in Q$ (see [R3] for some details), and the proof clearly follows.

We can now give (the $S$-generalization of) 
3.5 Proof of 3.1.2. By 3.3 and 3.4,

$$
\chi_{n}^{S}(B)=\sum_{\lambda \in H(m, 0 ; n)} m_{\lambda}^{S}(B) \cdot \chi_{\lambda},
$$

and $m_{\lambda}^{S}(B) \leq|\lambda|^{r_{1}}$ for some fixed $r_{1}$. Taking degrees, we obtain

$$
c_{n}^{S}(B) \leq n^{r_{1}} \sum_{\lambda \in H(m, 0 ; n)} d_{\lambda}
$$

The precise asymptotics of $\sum_{\lambda \in H(m, 0 ; n)} d_{\lambda}, n \rightarrow \infty$, is calculated in [R4]:

$$
\sum_{\lambda \in H(m, 0 ; n)} d_{\lambda} \underset{n \rightarrow \infty}{\simeq} C \cdot n^{r_{2}} \cdot m^{n}
$$

for some (explicit) constants $C$ and $r_{2}$. This clearly completes the proof.

\section{A Theorem of Razmyslov and multi-Alternating POlynomials}

We begin with an important theorem of Razmyslov. This theorem is essential for proving, in section 6 , the lower bounds for the codimensions.

4.1 Theorem [Ra, Thm. 12.1, p. 73]. Let B be a semisimple Lie algebra over an algebraically closed field of characteristic zero, and let $\operatorname{dim} B=m$. Let $B \subseteq U$ be an associative algebra generated by $B$, which is simple and with a non-zero center. Then there exist $k$ and a multilinear polynomial $f\left(x_{1}^{1}, \ldots, x_{m}^{1}, \ldots, x_{1}^{k}, \ldots, x_{m}^{k}\right)$, alternating in each $\left\{x_{1}^{i}, \ldots, x_{m}^{i}\right\}, 1 \leq i \leq k$, such that

1. $f$ does not vanish on $B$, and

2. for all $\bar{x}_{j}^{i} \in B, f\left(\bar{x}_{1}^{1}, \ldots, \bar{x}_{m}^{1}, \ldots, \bar{x}_{1}^{k}, \ldots, \bar{x}_{m}^{k}\right)$ belongs to the center of $U$.

We call the above $f(x)$ "multi-alternating" (or m.a.) and we introduce:

4.2 Definition. Let $m \cdot k \leq n$. Denote by $Q_{m, k, n} \subseteq V_{n}$ the subspace spanned by all polynomials that are alternating in $k$ disjoint subsets of variables $\left\{x_{1}^{i}, \ldots, x_{m}^{i}\right\} \subseteq$ $\left\{x_{1}, \ldots, x_{n}\right\}, 1 \leq i \leq k$. Then denote $Q_{m, k}=\bigcup_{n} Q_{m, k, n}$.

Here and in section 5 we deduce some properties of such polynomials.

Let $n=m k$ and let $f \in Q_{m, k, m k}$.

Clearly such a polynomial is a linear combination of "monomial" m.a. polynomials $p_{M}(x)$. Here $M(x)=M\left(x_{1}^{1}, \ldots, x_{m}^{1}, \ldots, x_{1}^{k}, \ldots, x_{m}^{k}\right)$ is a multilinear monomial, and

$$
p_{M}(x)=\sum_{\sigma^{(i)} \in S_{m}}(-1)^{\sigma^{(1)}} \cdots(-1)^{\sigma^{(k)}} \sigma^{(1)} \cdots \sigma^{(k)} M(x),
$$

where $\sigma^{(i)}$ permutes the variables $\left\{x_{1}^{i}, \cdots, x_{m}^{i}\right\}$.

Now, the right action of a permutation on a monomial is a position rearrangement of the variables. Hence there exists $\rho \in S_{m k}$ such that

$$
M(x)=\left(x_{1}^{1} \cdots x_{m}^{1} \cdot x_{1}^{2} \cdots x_{m}^{2} \cdots x_{1}^{k} \cdots x_{m}^{k}\right) \rho .
$$

It follows that

$$
p_{M}(x)=\left(s_{m}\left[x_{1}^{1}, \ldots, x_{m}^{1}\right] \cdots s_{m}\left[x_{1}^{k}, \ldots, x_{m}^{k}\right]\right) \rho,
$$

where $s_{m}\left[x_{1}, \ldots, x_{m}\right]$ is the standard polynomial. By taking linear combinations we obtain: 
4.3 Lemma. Let $f(x) \in Q_{m, k, m k}$ as above. Then there exists $a \in F S_{k m}$ such that

$$
f(x)=\left(s_{m}\left[x_{1}^{1}, \ldots, x_{m}^{1}\right] \cdots s_{m}\left[x_{1}^{k}, \ldots, x_{m}^{k}\right]\right) a .
$$

4.4 Remark. Note that $s_{m}\left[x_{1}, \ldots, x_{m}\right] \in I_{\left(1^{m}\right)}$. By Young's (or Pieri's) rule, in $F S_{k m}$,

$$
s_{m}\left[x_{1}^{1}, \ldots, x_{m}^{1}\right] \cdots s_{m}\left[x_{1}^{k}, \cdots, x_{m}^{k}\right] \in \bigoplus_{\substack{\lambda \vdash k m \\ \lambda_{1} \leq k}} I_{\lambda},
$$

where $I_{\lambda}$ is the two-sided ideal in $F S_{k m}$ corresponding to the partition $\lambda$.

We have thus proved

4.5 Proposition. In $F S_{k m}$

$$
Q_{m, k, m k} \subseteq \bigoplus_{\substack{\lambda \vdash k m \\ \lambda_{1} \leq k}} I_{\lambda}
$$

Note that if $\lambda \vdash k m$ and $\lambda_{1} \leq k$, then either $\lambda=\left(k^{m}\right)$, the $k \times m$ rectangle, or $\lambda_{1}^{\prime} \geq m+1$, where $\lambda^{\prime}$ is the conjugate partition of $\lambda$. Thus

4.6 Corollary. Let $A$ be an associative algebra and let $B \subseteq A$ be a subspace with $\operatorname{dim} B=m$. Let $p(x) \in Q_{m, k, m k}$, and write $p(x)=g(x)+r(x)$, where $g(x) \in I_{\left(k^{m}\right)}=I_{(k \times m)}$ and $r(x) \in \bigoplus_{\lambda \vdash k m, \lambda_{1}^{\prime}>m} I_{\lambda}$. Then $p(b)=g(b)$ for all $(b)=\left(b_{1}^{1}, \ldots, b_{m}^{k}\right), b_{j}^{i} \in B$.

Proof. Let $\lambda \vdash k m$ with $\lambda_{1}^{\prime} \geqq m$, and let $f(x) \in I_{\lambda}$. As in the proof of $3.4, f$ is a linear combination of polynomials, each with an alternating subset of at least $m+1$ variables. Hence $f(b)=0$, and so $r(b)=0$, for any such $(b)=\left(b_{1}^{1}, \ldots, b_{m}^{k}\right), b_{j}^{i} \in B$.

4.7 Remark. Let $\lambda \vdash n \geq k m$ be such that $\lambda_{m} \geq k$ (i.e. $\lambda$ contains the $k \times m$ rectangle). By (now) standard arguments it follows that $I_{\lambda} \subseteq Q_{m, k, n}$. (See for example [R3]).

\section{AN UPPER BOUND FOR THE LIE CODIMENSIONS}

By the Ado-Iwasawa theorem (see $\mathrm{B}, 6.2 .3]$ ) every finite dimensional Lie algebra $B$ over a field has a faithful finite dimensional representation. It follows that $B \subseteq$ $A^{(-)}$, where $A$ is a finite dimensional associative P.I. algebra. We now prove

5.1 Proposition. Let $B$ be a finite dimensional Lie algebra. Assume adB satisfies all the identities $f \equiv 0, f \in Q_{m+1, k}$, for some $m$ and $k$. Then $c_{n}^{L}(B) \leq C \cdot n^{r} \cdot m^{n}$ for some constants $C$ and $r$.

Proof. Let $\operatorname{dim} B=s$. By 3.3 and 3.4, $\chi_{n}^{L}(B)=\sum_{\lambda \in H(s, 0 ; n)} m_{\lambda} \chi_{\lambda}$ and $m_{\lambda} \leq|\lambda|^{r_{1}}$ for some $r_{1}$ and all $n$ 's and $\lambda$ 's. By 4.7 , if $\lambda_{m+1} \geq k$ then $I_{\lambda} \subseteq Q_{m+1, k}$; hence $I_{\lambda}$ are identities of $a d B$. It follows that $\chi_{n}^{L}(B)=\sum_{\substack{\lambda \in H(s, 0 ; n) \\ \lambda_{m+1} \leq k-1}} m_{\lambda} \chi_{\lambda}$, so

$$
c_{n}^{L}(B) \leq n^{r_{1}} \sum_{\substack{\lambda \in H(s, 0 ; n) \\ \lambda_{m+1} \leq k-1}} d_{\lambda}
$$

Denote $A(k, m, s, n)=\left\{\lambda \in H(s, 0 ; n) \mid \lambda_{m+1} \leq k-1\right\}$. The proof of 5.1 is complete once we prove 
5.2 Lemma. There exist constants $C$ and $r$ such that $\sum_{\lambda \in A(k, m, s, n)} d_{\lambda} \leq C \cdot n^{r}$. $m^{n}$.

Proof. Let $\lambda \in A(k, m, s, n)$ and denote $\bar{\lambda}=\left(\lambda_{1}, \cdots, \lambda_{m}\right)$ and $u=u(\lambda)=\lambda_{m+1}+$ $\cdots+\lambda_{s}$. Since $k-1 \geq \lambda_{m+1} \geq \cdots \geq \lambda_{s}, u \leq(s-m) \cdot(k-1)$. By the branching rule, $\chi_{\lambda}$ appears in $\psi=\chi_{\bar{\lambda}} \hat{\otimes} \underbrace{\chi_{(1)} \hat{\otimes} \cdots \hat{\otimes} \chi_{(1)}}_{u}$; hence $d_{\lambda}=\operatorname{deg} \chi_{\lambda} \leq \operatorname{deg} \psi$.

Now $\operatorname{deg}\left(\chi_{\mu} \hat{\otimes} \chi_{(1)}\right)=(|\mu|+1) \operatorname{deg} \chi_{\mu}$, so $d_{\lambda} \leq \operatorname{deg} \psi \leq n^{u} d_{\bar{\lambda}}$. Thus

$$
\sum_{\lambda \in A(k, m, s ; n)} d_{\lambda} \leq \sum_{u=0}^{(s-m)(k-1)} n^{u} \sum_{\mu \in H(m, 0 ; n-u)} d_{\mu} .
$$

By [R4] we have $\sum_{\mu \in H(m, 0 ; n)} d_{\mu} \simeq C_{2} n^{r_{2}} m^{n}$ for some (explicit) constants $C_{2}$ and $r_{2}$, and the proof of 5.2 follows.

This also completes the proof of Proposition 5.1.

$$
\text { 6. } \operatorname{Inv}^{L}(B)=\operatorname{dim} B \text { WHEN } F=\bar{F} \text { AND } B \text { IS SIMPLE }
$$

Here we need to apply 4.1 ; hence we assume that $F$ is algebraically closed. We prove:

6.1 Theorem. Let $F=\bar{F}$ (i.e., $F$ is algebraically closed) and let $B$ be a simple Lie algebra, $\operatorname{dim} B=m$. Then there exist constants $C_{1}, C_{2}>0, r_{1}$ and $r_{2}$ such that

$$
C_{2} \cdot n^{r_{2}} \cdot m^{n} \leq c_{n}^{L}(B) \leq C_{1} \cdot n^{r_{1}} \cdot m^{n}
$$

for all $n$. In particular, $\lim _{n \rightarrow \infty} \sqrt[n]{c_{n}^{L}(B)}=\operatorname{Inv}(B)=m=\operatorname{dim} B$.

Proof. Note that by simplicity $B \cong a d B \subseteq \operatorname{End}(B)^{(-)}$; hence Theorem $3.1 .2 \mathrm{im}$ plies the upper bound.

To prove the lower bound we apply 4.1. Note that by simplicity adB generates the associative (enveloping) algebra $\operatorname{End}(B)=U$, and $U$ has a non-trivial center. By 4.1 (with $a d B$ replacing $B$ ) there exists a multilinear polynomial $f\left(x_{1}^{1}, \ldots, x_{m}^{1}, \ldots, x_{1}^{k}, \ldots, x_{m}^{k}\right)$, alternating in each $\left\{x_{1}^{i}, \ldots, x_{m}^{i}\right\}, 1 \leq i \leq k$, and $f$ is a central-non-identity of $a d B$. Thus there exist $b_{j}^{i}, a \in B$ such that

$$
f\left(a d b_{1}^{1}, \ldots, a d b_{m}^{1}, \ldots, a d b_{1}^{k}, \ldots, a d b_{m}^{k}\right)(a)=\alpha a \neq 0, \quad 0 \neq \alpha \in F .
$$

Given $n$, write $n-1=t k m+q, 0 \leq q \lesseqgtr k m$. Also denote

$$
f\left(x_{1}^{1}, \ldots, x_{m}^{1}, \ldots, x_{1}^{k}, \ldots, x_{m}^{k}\right)=f\left(x_{1}, \ldots, x_{k m}\right) .
$$

Let

$$
g\left(x_{1}, \ldots, x_{n-1}\right)=g_{t k m}(x) \cdot x_{t k m+1} \cdots x_{n-1},
$$

where $g_{t k m}(x)=f\left(x_{1}, \ldots, x_{k m}\right) f\left(x_{k m+1}, \ldots, x_{2 k m}\right) \cdots f\left(x_{(t-1) k m+1}, \ldots, x_{t k m}\right)$. It easily follows that $g\left(x_{1}, \ldots, x_{n-1}\right)$ is not an identity of $a d B$.

Clearly, $g_{t k m}(x)$ is alternating in $t k$ disjoint sets of $m$ variables each. As in 4.6, we can decompose $g_{t k m}=g_{R}+g^{*}$, where $g_{R} \in I_{\left((t k)^{m}\right)}$ and $g_{t k m}(x)=g_{R}(x)$ on $a d B$. Thus $g_{R}$ is a non-identity on $a d B$. Therefore, by $2.3, c_{n}^{L}(B) \geq d_{R}=d_{\left((t k)^{m}\right)}$.

Note that, as $n \rightarrow \infty, n \simeq t k m=|R|$, where $R$ is the partition $R=\left((t k)^{m}\right)$. The proof of 6.1 now follows, since by [R4, F.1.1], $d_{R} \underset{n \rightarrow \infty}{\simeq} u \cdot n^{v} \cdot m^{n}$ for some (explicit) constants $u$ and $v$. 
We remark that 6.1 can be generalized to the following:

6.2 Theorem. Let $B$ be a finite dimensional Lie algebra. Let $H$ be a semisimple Lie subalgebra, $H \subseteq B$, such that, as adH modules, $B$ has at least one faithful irreducible submodule $M \subseteq B$. Let $\operatorname{dim} H=m$. Then $c_{n}^{L}(B) \geq C \cdot n^{r} \cdot m^{n}$ for some constants $C>0$ and $r$.

Proof. Denote $a d: H \rightarrow a d_{M}(H) \subseteq \operatorname{End}(M)$. By faithfulness, $H \cong a d_{M}(H)$, and by simplicity, $a d_{M}(H)$ generates $\operatorname{End}(M)$. The rest of the proof is identical to that of 6.1 .

\section{A characterization of simple Lie algebras, $F=\bar{F}$}

Theorem 6.1 generalizes to:

7.1 Theorem. Let $F=\bar{F}$. Let $B$ be a finite dimensional semisimple Lie algebra: $B=\bigoplus_{i} B_{i}, B_{i}$ simple. Let $m_{0}=\max _{i}\left\{m_{i}\right\}, m_{i}=\operatorname{dim} B_{i}$. Then

$$
C_{2} n^{r_{2}} m_{0}^{n} \leq c_{n}^{L}(B) \leq C_{1} n^{r_{1}} m_{0}^{n}
$$

for some $C_{1}, C_{2}>0, r_{1}$ and $r_{2}$. In particular, $\operatorname{Inv}^{L}(B)=m_{0}$.

Proof. First, $B_{i} \subseteq B$; hence $c_{n}^{L}\left(B_{i}\right) \leq c_{n}^{L}(B)$, and 6.1 (for $B_{i}$ ) implies the lower bound.

Conversely, $a d B_{i}$ satisfies $f \equiv 0$ for all $f \in Q_{m_{i}+1,1}$, hence for all $f \in Q_{m_{0}+1,1}$. Therefore $B$ satisfies all $f \in Q_{m_{0}+1,1}$, and the upper bound follows from 5.1 (note that $B \cong a d B$ here).

7.2 Lemma. Let $B$ be a finite dimensional Lie algebra which is not semisimple. Denote $\operatorname{dim} B=m$. Then $c_{n}^{L}(B) \leq C \cdot n^{r} \cdot(m-1)^{n}$ for some $c, r$.

Proof. It is well known that such a $B$ contains an abelian ideal $0 \neq N \subset B$ with $[N, N]=0$. Denote $\bar{B}=B / N$. We clearly have $\operatorname{dim}(a d \bar{B}) \leq \operatorname{dim} \bar{B}=\bar{m} \leq m-1$. This implies that $a d \bar{B}$ satisfies all $f \in Q_{\bar{m}+1,1}$, and we show that $a d B$ satisfies all $f \in Q_{\bar{m}+1,2}$.

Indeed, complete a basis of $a d N(\subseteq a d B)$ to a basis of $a d B$. In any $\bar{m}+1$ distinct elements from that basis there is at least one element from adN. Let $f\left(x_{1}^{1}, \ldots, x_{\bar{m}+1}^{1}, x_{1}^{2}, \ldots, x_{\bar{m}+1}^{2}, y_{1}, y_{2}, \ldots\right) \in Q_{\bar{m}+1,2}$ be alternating in the two subsets $\left\{x_{1}^{i}, \ldots, x_{\bar{m}+1}^{i}\right\}, i=1,2$. By multilinearity, substitutions from $a d B$ can be taken from that basis of $a d B$. A repetition in an alternating subset yields zero. Thus, in any "basis" substitution with no such repetitions, there are at least two elements from $a d N$, and hence the corresponding value of $f$ is in $[N, N]=0$. The proof now follows from 5.1.

Thus, we obtain the following characterization of the simplicity of $B$ in terms of $\operatorname{Inv} v^{L}(B)$ :

7.3 Theorem. Let $F=\bar{F}$, and let $B$ be a finite dimensional Lie algebra. Then $B$ is simple if and only if $\operatorname{Inv} v^{L}(B)$ exists (i.e. $\overline{\operatorname{Inv}}^{L}(B)=\underline{\operatorname{Inv}}^{L}(B)$ ) and $\operatorname{Inv} v^{L}(B)=$ $\operatorname{dim} B$. Moreover, if $B$ is not simple then $\overline{\operatorname{Inv}}^{L}(B) \leq \operatorname{dim} B-1$.

Proof. If $B$ is simple, $\operatorname{Inv}^{L}(B)=\operatorname{dim} B$ by 6.1 . If $B$ is not simple, then either it is semisimple or $B$ contains an abelian ideal $0 \neq N \subseteq B$.

Case 1: $\quad B$ is semisimple (but not simple); hence $B=\bigoplus_{i} B_{i}$ as in 7.1, and $\operatorname{dim} B_{i} \lesseqgtr \operatorname{dim} B$ for all $i$. By 7.1, $\overline{\operatorname{Inv}}^{L}(B) \lesseqgtr \operatorname{dim} B-1$. 
Case 2: $\quad$ Let $0 \neq N \subseteq B$ be an abelian ideal: $[N, N]=0$. By $7.2, \overline{\operatorname{Inv}}^{L}(B) \supsetneqq$ $\operatorname{dim} B-1$ again.

\section{The general case, $\operatorname{Char}(F)=0$}

Simple Lie algebras in characteristic zero are studied and classified, for example, in [Jac Chap.10]. Here is a short summary of the most basic facts:

If $B$ is a simple Lie algebra over $F$, then its centroid (i.e., the centralizer of the adjoint action) is a field $E \supseteq F$, and $B$ is central if $E=F$. Let $\bar{F}$ denote the algebraic closure of $F$; then $B \otimes_{F} \bar{F}$ is a direct sum of $[E: F]$ copies of a fixed simple Lie $\bar{F}$-algebra.

Let $F \subseteq K$ be a field extension, $W$ an $F$ vector space, and $W_{K}=W \otimes_{F} K$.

If $B$ is a semisimple Lie algebra over $F$, it follows that $B_{\bar{F}}$ is also semisimple.

It is well known that field extensions do not affect multilinear identities; hence the codimensions: $c_{n}^{L}(B)=c_{n}^{L}\left(B_{K}\right)$. Thus $\overline{\operatorname{Inv}}^{L}(B)=\overline{\operatorname{Inv}}^{L}\left(B_{K}\right), \underline{\operatorname{Inv}}^{L}(B)=$ $\underline{\operatorname{Inv}}^{L}\left(B_{K}\right)$, and hence $\operatorname{Inv} v^{L}(B)=\operatorname{Inv} v^{L}\left(B_{K}\right)$.

Now let $K=\bar{F}$. The above remarks, together with 7.3 , imply:

8.1 Theorem. Let $F$ be a field of characteristic zero and let $B$ be a finite dimensional Lie algebra over $F$. Then $B$ is central simple if and only if $\operatorname{Inv}^{L}(B)$ exists and $\operatorname{Inv}^{L}(B)=\operatorname{dim} B$.

Similarly:

8.2 Theorem. Let char $(F)=0$, and let $B$ be a finite dimensional Lie algebra over $F$. If $B$ is simple, then $\operatorname{Inv}^{L}(B)$ exists, is a positive integer which divides $\operatorname{dim} B$, and

$$
\frac{\operatorname{dim} B}{\operatorname{Inv}^{L}(B)}=[\text { centroid }(B): F]
$$

Proof. We have $B \otimes \bar{F}=B_{1} \oplus \cdots \oplus B_{j}$, where $j=[$ Centroid $(B): F]$ and $B_{1} \cong \ldots \cong B_{j}$ are $\bar{F}$-simple. Clearly, $\operatorname{dim} B_{1}=\frac{\operatorname{dim} B}{j}$, and by $7.1, \operatorname{Inv}^{L}(B)=$ $\operatorname{Inv} v^{L}\left(B_{\bar{F}}\right)=\operatorname{dim} B_{1}$.

In the semisimple case, the above remarks and 7.1 imply

8.3 Theorem. Let char $(F)=0$, and let $B$ be a finite dimensional semisimple Lie algebra over $F$. Then $\operatorname{Inv}^{L}(B)$ exists and is an integer.

In fact, let $\bar{F}$ be the algebraic closure of $F$ and write $B_{\bar{F}}=\bigoplus_{i} \widetilde{B}_{i}$, where the $\widetilde{B}_{i}$ 's are simple (over $\left.\bar{F}\right)$. Let $m_{0}=\max _{i}\left\{\operatorname{dim} \widetilde{B}_{i}\right\}$.

Then $\operatorname{Inv}^{L}(B)=\operatorname{Inv}^{L}\left(B_{\bar{F}}\right)=m_{0}$.

We also have

8.4 Proposition. Let $B$ be a finite dimensional Lie algebra over $F$. If $B$ is not simple, then $\overline{\operatorname{Inv}}^{L}(B) \leq \operatorname{dim}_{F} B-1$.

Proof. If $B$ is not simple, it easily follows that $B_{\bar{F}}$ is not simple. Hence, by 7.3 ,

$$
\overline{\operatorname{Inv}}^{L}(B)=\overline{\operatorname{Inv}}^{L}\left(B_{\bar{F}}\right) \leq \operatorname{dim}_{\bar{F}}\left(B_{\bar{F}}\right)-1=\operatorname{dim}_{F} B-1 .
$$


We conclude this section with the following corollary.

Let $G$ be a finite-dimensional semisimple Lie algebra, $F\left[t, t^{-1}\right]$ the ring of Laurent polynomials and $\tilde{G}=G \otimes F\left[t, t^{-1}\right]+\langle z\rangle$ a non-twisted affine Kac-Moody algebra corresponding to $G$.

8.5 Corollary. If $G$ is a finite-dimensional semisimple Lie algebra and $\tilde{G}$ a nontwisted affine Kac-Moody algebra corresponding to $G$, then $\operatorname{Inv}^{L}(\tilde{G})=\operatorname{Inv}^{L}(G)=$ $k$, where $k$ is a positive integer.

The proof follows immediately from the previous theorem and from the construction of the finite-dimensional Lie algebra $\bar{G}$ such that $\operatorname{var} \tilde{G}=\operatorname{var} \bar{G}$ (see [Z]). The corollary may also be proved directly, since $\tilde{G}$ is a central extension of $G \otimes F\left[t, t^{-1}\right]$ and this last has the same identities of $G$. Also, it is not difficult to show that if $A$ is a central extension of $B$, then $\operatorname{Inv}^{L}(A)=\operatorname{Inv} v^{L}(B)$.

\section{Some SPeCial Jordan AlgEbras}

In this section we compute the exponential growth of some special Jordan algebras of small dimension. Define $\left\{x_{1},\left\{x_{2}, \ldots,\left\{x_{n-1}, x_{n}\right\}, \ldots\right\}\right\}=\left\{x_{1}, \ldots, x_{n}\right\}$, right normed Jordan monomials. In this case the map

$$
\phi: x_{\sigma(1)} \cdots x_{\sigma(n)} \rightarrow\left\{x_{\sigma(1)} \cdots x_{\sigma(n)}, x_{0}\right\}
$$

induces an $F S_{n}$-module monomorphism $V_{n} \rightarrow V_{n+1}^{S}$, and the analogue of 2.2 still holds, where the $V_{n+1}^{S}$ are now multilinear Jordan polynomials in $x_{0}, x_{1}, \ldots, x_{n}$.

Let $M_{n}(F)$ be the algebra of $n \times n$ matrices over the field $F$. We denote by $M_{n}(t)^{+}$and $M_{n}(s)^{+}$the Jordan subalgebras of symmetric elements of $M_{n}(F)$ under the transpose and symplectic involution respectively. We have

9.1 Theorem. 1. For $k=2,3, \quad \operatorname{Inv} v^{J}\left(M_{k}(t)^{+}\right)=\operatorname{dim} M_{k}(t)^{+}=k(k+1) / 2$.

2. For $k=2,4, \quad \operatorname{Inv}^{J}\left(M_{k}(s)^{+}\right)=\operatorname{dim} M_{k}(s)^{+}=k(k-1) / 2$.

Proof. By 3.1, $\overline{\operatorname{Inv}}^{J}\left(M_{k}(*)^{+}\right) \leq \operatorname{dim} M_{k}(*)^{+}$where $*=t$ or $s$.

Let $L_{x}$ denote the left Jordan multiplication by $x$. For $k=2$ take $a_{1}=e_{11}-$ $e_{22}, a_{2}=e_{11}+e_{22}, a_{3}=e_{12}+e_{21}$ as basis of $M_{2}(t)^{+}$, let $n=3 l+t, 0 \leq t<3$, and let

$$
f\left(x_{1}, \ldots, x_{n}\right)=s_{3}\left(x_{1}, x_{2}, x_{3}\right) s_{3}\left(x_{4}, x_{5}, x_{6}\right) \cdots s_{3}\left(x_{3 l-2}, x_{3 l-1}, x_{3 l}\right) x_{3 l+1} \cdots x_{3 l+t},
$$

where $s_{3}$ denotes the standard polynomial of degree three. It can be checked that $s_{3}\left(L_{a_{1}}, L_{a_{2}}, L_{a_{3}}\right)^{k} L_{a_{1}}^{t}\left(a_{1}\right) \neq 0$. Thus $f\left(x_{1}, \ldots, x_{n}\right)$ is not an identity on $\left\{L_{a} \mid\right.$ $\left.a \in M_{2}(t)\right\}$ and, by $2.3, c_{n+1}^{J}\left(M_{2}(t)^{+}\right) \geq d_{\lambda}$, where $\lambda=\left(k^{3}\right)$. It follows that $\underline{\operatorname{Inv}}^{J}\left(M_{2}(t)^{+}\right) \geq 3$, and this, combined with the above, gives $\operatorname{Inv} v^{J}\left(M_{2}(t)^{+}\right)=3$.

To prove (1) for $k=3$, let $\mu=\left(2^{6}\right) \vdash 12$ and let $T_{\mu}$ be the following tableau:

\begin{tabular}{|r|r|}
\hline 1 & 2 \\
\hline 3 & 6 \\
\hline 4 & 7 \\
\hline 5 & 8 \\
\hline 9 & 11 \\
\hline 10 & 12 \\
\hline
\end{tabular}

Let $f\left(x_{1}, \ldots, x_{12}\right)=e_{T_{\mu}}\left(x_{1}, \ldots, x_{12}\right)$ be the corresponding (associative) polynomial. 
For every natural $n$ write $n=12 l+t$, where $0 \leq t<12$, and let

$$
g\left(x_{1}, \ldots, x_{n}\right)=f\left(x_{1}, \ldots, x_{12}\right) \cdots f\left(x_{12(l-1)+1}, \ldots, x_{12 l}\right) x_{12 l+1} \cdots x_{n} .
$$

Consider the basis $\left\{a_{1}=e_{11}, a_{2}=e_{22}, a_{3}=e_{33}, a_{4}=e_{12}+e_{21}, a_{5}=e_{13}+\right.$ $\left.e_{31}, a_{6}=e_{23}+e_{32}\right\}$ of $M_{3}(t)^{+}$and the substitution $\phi: x_{1}, x_{2} \rightarrow L_{a_{1}}, x_{3}, x_{6} \rightarrow$ $L_{a_{2}}, x_{4}, x_{7} \rightarrow L_{a_{3}}, x_{5}, x_{8} \rightarrow L_{a_{4}}, x_{9}, x_{11} \rightarrow L_{a_{5}}, x_{10}, x_{12} \rightarrow L_{a_{6}}$. By a computer calculation it can be checked that $\phi(f)\left(a_{4}\right)=\alpha a_{4}$ for some non-zero integer $\alpha$. It follows that $g\left(x_{1}, \ldots, x_{n}\right)$ is not an identity on $\left\{L_{a} \mid a \in M_{3}(t)\right\}$, and the conclusion follows as before.

2 ) is proved similarly.

\section{REFERENCES}

[B] Yu. A. Bahturin, Identical Relations in Lie Algebras, VNU Science Press, Utrecht, 1987. MR 88f: 17032

[BMR] Yu. A. Bahturin, S. P. Mischenko and A. Regev, On the Lie and associative codimension growth, preprint.

[BR] A. Berele and A. Regev, Applications of hook diagrams to P.I. algebras, J. Algebra 82 (1983), 559-567. MR 84g:16012

[Jac] N. Jacobson, Lie Algebras, Interscience Publishers, 1962. MR 26:1345

$[\mathrm{M}] \quad$ S. P. Mischenko, Growth of varieties of Lie algebras, Uspekhi Matem. Nauk. 45 (1990), 25-45; English transl., Russian Math. Surveys 45 (1990), no. 6, 27-52. MR 92g:17003

[MP] S. P. Mischenko and V. M. Petrogradsky, Exponents of varieties of Lie algebras with a nilpotent commutator subalgebra, Comm. Algebra 27 (1999), 2223-2230. CMP 99:11

[P] V. M. Petrogradsky, Growth of polynilpotent varieties of Lie algebras and fast increasing entire functions, Mat. Sb. 188 (1997), no. 6, 119-138; English transl., Sb. Math. 188 (1997), 913-931. MR 99a:17008

[Ra] Yu. P. Razmyslov, Identities of algebras and their representations, Transl. Math. Monogr. vol. 138, Amer. Math. Soc., Providence, RI, 1994. MR 95i:16022

[R1] A. Regev, Existence of identities in $A \otimes B$, Israel J. Math 11 (1972), 131-152. MR 47:3442

[R2] A. Regev, The representations of $S_{n}$ and explicit identities for P.I. algebras, J. Algebra 51 (1978), 25-40. MR 57:4745

[R3] A. Regev, The polynomial identities of matrices in characteristic zero, Comm. Algebra 8 (1980), 1417-1467. MR 83c:15015

[R4] A. Regev, Asymptotic values for degrees associated with stripes of Young diagrams, Adv. Math. 41 (1981), 115-136. MR 82h:20015

[V] I. B. Volichenko, Bases of a free Lie algebra modulo T-ideals, Dokl. Akad. Nauk BSSR 24 (1980), n. 5, 400-403. (Russian) MR 81h:17007

[ZM] M. V. Zaicev and S. P. Mishchenko, Varieties of Lie subalgebras of polynomial growth, Uspekhi Matem. Nauk 52 (1997), no. 2, 165-166; English transl., Russian Math. Surveys 52 (1997), 432-433. CMP 98:04

[Z] M. V. Zaicev, Identities of affine Kac-Moody algebras, Vestnik Moskov. Univ. Ser. I Mat. Mekh. 1996, no. 2, 33-36; English transl., Moscow Univ. Math. Bull. 51 (1996), no. 2, 29-31. CMP 98:05

Department of Mathematics, University of Palermo, Via Archirafi 34, 90123 Palermo, ITALY

E-mail address: a.giambruno@unipa.it

Department of Theoretical Mathematics, The Weizmann Institute of Science, Rehovot 76100, Israel and The Pennsylvania State University, University Park, PennSYLVANIA 16802

E-mail address: regev@wisdom.weizmann.ac.il

E-mail address: regev@math.psu.edu

Department of Mechanics and Mathematics, Moscow State University, Moscow 119899, Russia

E-mail address: zaicev@nw.math.msu.su 